\title{
Precise Robustness Analysis of Time Petri Nets with Inhibitor Arcs*
}

\author{
Étienne André, Giuseppe Pellegrino*^, and Laure Petrucci \\ Université Paris 13, Sorbonne Paris Cité, LIPN \\ F-93430, Villetaneuse, France
}

\begin{abstract}
Quantifying the robustness of a real-time system consists in measuring the maximum extension of the timing delays such that the system still satisfies its specification. In this work, we introduce a more precise notion of robustness, measuring the allowed variability of the timing delays in their neighbourhood. We consider here the formalism of time Petri nets extended with inhibitor arcs. We use the inverse method, initially defined for timed automata. Its output, in the form of a parametric linear constraint relating all timing delays, allows the designer to identify the delays allowing the least variability. We also exhibit a condition and a construction for rendering robust a non-robust system.
\end{abstract}

Keywords: Time Petri nets, Quantitative robustness, Parameter synthesis

\section{Introduction}

Formalisms for modelling real-time systems, such as time Petri nets [Mer74] or timed automata [AD94], have been extensively used in the past decades, and led to useful and efficient implementations. Time Petri nets (TPNs for short) are an extension of Petri nets where firing conditions are given in the form of intervals $[a, b]$. Each transition can only fire at least $a$ time units and at most $b$ time units after it is enabled. ITPNs extend TPNs with inhibitor arcs, i.e. arcs that disable their outgoing transition if their incoming place is not empty.

However, these formalisms allow for modelling in theory delays arbitrarily close (or even equal) to zero; this implies that the real system must be arbitrarily fast, which may be unrealistic in practice, where response times may not be neglected. These formalisms also allow for simultaneous occurrence of events, which may not be realistic in practice either, due to slightly different clock rates of several processors. And similarly, they allow for arbitrary precision, which is unrealistic: For example, a system where some component performs an action for e.g. 2 seconds can be implemented with a delay greater but very close to 2 (e.g. $2.0001 \mathrm{~s}$ ), in which case the formal guarantee may not hold anymore.

\footnotetext{
* This is the author version of the paper of the same name accepted for publication at FORMATS 2013. The final publication is available at www.springer.com.

** This work is partially supported by an Erasmus grant.
} 
The implementation in practice of a real-time system (modelled, e.g. by an ITPN) can lead in particular to two kinds of undesired consequences: the occurrence of behaviours that were proven impossible in theory, and the unlikely occurrence of behaviours that were proven possible in theory.

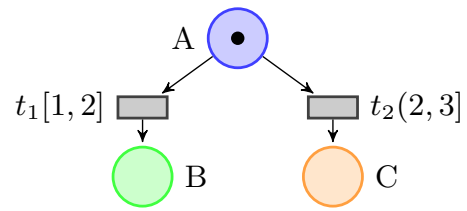

(a) Example of undesired reachability

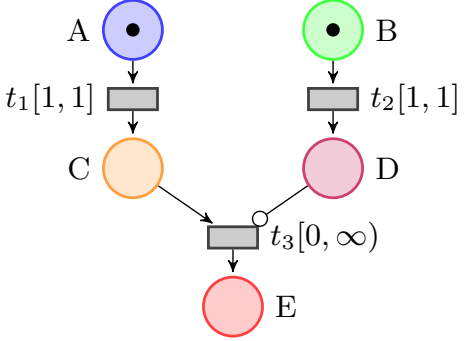

(b) Example of unlikely reachability

Fig. 1: Examples of non-robust ITPNs

Consider the simple TPN in Fig. 1a (from [AHJR12]). According to the semantics of TPNs (e.g. defined in [TLR09]), place $\mathrm{C}$ is unreachable, that is, there exists no reachable marking such that the number of tokens in $\mathrm{C}$ is greater than 0 . Indeed, starting from marking A (i.e. a marking with 1 token in place A), $t_{1}$ can fire anytime between 1 and 2 time units after the system start. At time $2, t_{1}$ must fire if it has not yet fired, because its associated interval is about to expire and no other transition is firable $\left(t_{2}\right.$ will be firable right after time 2$)$. Hence, $\mathrm{C}$ is unreachable. Now suppose that the upper bound of the firing interval of $t_{1}$ is increased, even by an infinitesimal duration. Then, $t_{2}$ is firable immediately after time 2 , and $\mathrm{C}$ can be reached in some executions.

Now consider the ITPN in Fig. 1b. According to the semantics of ITPNs, place $\mathrm{E}$ is reachable. Indeed, starting from a marking $\mathrm{AB}$ (i.e. a marking with 1 token in place $\mathrm{A}$ and 1 token in place $\mathrm{B}), t_{1}$ can fire at time 1 , giving marking $\mathrm{CB}$. Then, after a null duration, $t_{3}$ can fire due to the absence of token in $\mathrm{D}$. This sequence of transitions is unlikely to happen in practice due to delays exactly equal to zero; if the bounds of $t_{1}$ or the lower bound of $t_{3}$ become slightly larger, or the bounds of $t_{2}$ becomes slightly smaller, $\mathrm{E}$ becomes unreachable.

In this work, we use techniques based on parameter synthesis to compute a precise quantitative analysis of the admissible variability of the timing bounds of an ITPN with respect to linear-time properties. We use PITPNs, that is extensions of ITPNs where timing bounds are parameters, i.e. unknown constants. Our contributions are as follows:

1. We define the notion of covering constraint for parametric time Petri nets with inhibitor arcs (PITPNs), and characterise it; 
2. We extend the inverse method to PITPNs (initially defined in the setting of parametric timed automata [AS13]), and prove that it preserves linear-time properties, based on the notion of covering constraint; and

3. We exploit the constraint output to obtain a precise quantitative measure of the system robustness for linear-time properties.

Given in the form of a constraint on the timing bounds seen as parameters, our robustness condition allows a designer (i) to relate the variability of the timing bounds with each other, (ii) to exhibit the critical timing bounds that do not allow any variability, and (iii) to render a system robust under certain conditions.

Related Work. Robustness in the setting of timed automata has received much attention in the past decade (see [Mar11] for a survey). Most previous works (see e.g. $\left[\mathrm{BLM}^{+} 11\right.$, Mar11,JR11,AHJR12,Tra12,BMS12]) consider that all timing constraints can be enlarged by a single very small (but positive) variation $\Delta$. This robustness condition considers a unique positive parameter $\Delta$; hence, roughly speaking, the robustness is guaranteed as long as the different clocks remain in intervals $[a-\Delta, b+\Delta]$ instead of $[a, b]$. In a geometrical context, the admissible variability can be seen as a simple hypercube (called " $\Delta$-cube" from now on) in $2 * n$ dimensions, with $n$ the number of timing constraints. In contrast, we give a precise measure of the robustness, by considering possible local variations of each lower and upper bound of the firing intervals of a time Petri net. This is given in the form of a polyhedron in $2 * n$ dimensions, where $n$ is the number of transitions. Hence, each bound can vary independently of the others. Our approach has the following advantages: (1) it identifies the most critical interval bounds, and helps the designer in tuning them (when possible) so that the system becomes robust; (2) it relates bounds in a parametric way, identifying bounds that should, for example, remain smaller than others; (3) it also outputs a constraint even when some bounds cannot tolerate any variation, whereas $\Delta$-based approaches would just classify the system as non-robust (i.e. synthesise a $\Delta=0$ ). Since parameter synthesis is undecidable for PITPNs [TLR09], our algorithm may not terminate in the general case; however, we give sufficient termination conditions for subclasses of PITPNs.

In [BMS12], it is shown that parameterised robust reachability in timed automata is decidable, again for a single $\Delta$. In [JR11], computing the greatest acceptable variation $\Delta$ is proven decidable for flat timed automata with progressive clocks. In [Tra12], a counter-example refinement approach is used with parametric techniques to evaluate the greatest acceptable variation $\Delta$ for parametric timed automata (although not decidable in the general case). These works share similarities with ours in the problem addressed and in the use of parametric techniques. However, beyond the fact that these works consider (a restriction of) timed automata whereas we consider (an extension of) time Petri nets, the main difference lies in the number of dimensions, since they all consider a simple $\Delta$.

Recent work also considered robustness issues in time Petri nets. In [AHJR12], the quantification of robustness is performed by considering that the firing intervals can be enlarged by a (positive) parameter. Two problems are considered: 
the robust boundedness of the net (a bounded net remains bounded even in presence of small time variations) and the robust untimed language preservation (the untimed language remains preserved in presence of small time variations). Our work is close to [AHJR12], with notable differences. First, we use here a technique based on parameter synthesis. Second, we give a condition for trace preservation, where traces are defined as alternating markings and actions. Hence, the robustness condition in our work is different from the boundedness and language preservation of [AHJR12]. Last but not least, the robustness condition in [AHJR12] again considers a unique positive parameter $\Delta$, whereas we compute a polyhedron in $2 * n$ dimensions. In [AHJ $\left.{ }^{+} 12\right]$, a more general notion of robustness is used for time Petri nets, that includes not only a robustness with respect to time, but also with constraints on the resources (e.g. memory), scheduling schemes (in a multi-processor environment) and possible system failures.

Outline. Section 2 recalls PITPNs and related results. In Section 3, we introduce and characterise covering constraints. In Section 4, we introduce the inverse method for PITPNs and prove its correctness. In Section 5, we exhibit a precise quantitative measure of the system robustness, and use it to turn some nonrobust systems robust. We give directions of future research in Section 6 .

\section{Preliminaries}

We denote by $\mathbb{N}, \mathbb{Q}_{+}$and $\mathbb{R}_{+}$the sets of non-negative integers, non-negative rational and non-negative real numbers, respectively.

\subsection{Firing Times, Parameters and Constraints}

Throughout this paper, we assume a set $\left\{\theta_{1}, \theta_{2}, \ldots\right\}$ of firing times. A firing time is a variable with value in $\mathbb{R}_{+}$, encoding the time remaining before a given transition fires. In the following, $\Theta$ will denote a finite set $\left\{\theta_{1}, \ldots, \theta_{H}\right\}$ of firing times, for some $H \in \mathbb{N}$. A firing time valuation is a function $\nu: \Theta \rightarrow \mathbb{R}_{+}^{H}$ assigning a non-negative real value with each firing time.

We also assume a set $\left\{\lambda_{1}, \lambda_{2}, \ldots\right\}$ of parameters, i.e. unknown constants. In the following, $\Lambda=\left\{\lambda_{1}, \ldots, \lambda_{l}\right\}$ denotes a finite set of parameters for some $l \in \mathbb{N}$. A parameter valuation $\pi$ is a function $\pi: \Lambda \rightarrow \mathbb{R}_{+}$assigning with each parameter a value in $\mathbb{R}_{+}$. A valuation $\pi$ can be seen as a point $\left(\pi\left(\lambda_{1}\right), \ldots, \pi\left(\lambda_{l}\right)\right)$.

Constraints are defined as a set of inequalities. A (linear) inequality over $\Theta$ and $\Lambda$ is $l t \prec l t^{\prime}$, where $\prec \in\{<, \leq\}$, and $l t, l t^{\prime}$ are two linear terms of the form $\sum_{1 \leq i \leq N} \alpha_{i} z_{i}+d$ where $z_{i} \in \Theta \cup \Lambda, \alpha_{i} \in \mathbb{Q}_{+}$, for $1 \leq i \leq N$, and $d \in \mathbb{Q}_{+}$. We define similarly inequalities over $\Theta$ (resp. $\Lambda$ ). A constraint is a conjunction of inequalities. In particular, a constraint over the parameters can be seen as a polyhedron in $l$ dimensions. We denote by $\mathcal{L}(\Lambda)$ the set of all constraints over the parameters. In the sequel, $J$ denotes an inequality over the parameters, $E$ a constraint over the firing times, $K$ a constraint over the parameters, and $D$ a 
constraint over firing times and parameters. Often, given a PITPN transition $t_{i}$, we will denote its parametric lower and upper bounds by $\lambda_{i}^{-}$and $\lambda_{i}^{+}$, respectively.

Given an inequality $J$ of the form $l t<l t^{\prime}$ (respectively $l t \leq l t^{\prime}$ ), the negation of $J$, denoted by $\neg J$, is the inequality $l t^{\prime} \leq l t$ (respectively $l t^{\prime}<l t$ ).

Given a constraint $E$ and a firing time valuation $\nu, \llbracket E \rrbracket_{\nu}$ denotes the expression obtained by replacing each firing time $\theta$ in $E$ with $\nu(\theta)$. A firing time valuation $\nu$ satisfies constraint $E$ (denoted by $\nu \models E$ ) if $\llbracket E \rrbracket_{\nu}$ evaluates to true.

Given a parameter valuation $\pi$ and a constraint $D, \llbracket D \rrbracket_{\pi}$ denotes the constraint over $\Theta$ obtained by replacing each parameter $\lambda$ in $D$ with $\pi(\lambda)$. Likewise, given a firing time valuation $\nu, \llbracket \llbracket D \rrbracket_{\pi} \rrbracket_{\nu}$ denotes the expression obtained by replacing each firing time $\theta$ in $\llbracket D \rrbracket_{\pi}$ with $\nu(\theta)$. We say that a parameter valuation $\pi$ satisfies a constraint $D$, denoted by $\pi \models D$, if the set of firing time valuations that satisfy $\llbracket D \rrbracket_{\pi}$ is non-empty.

A parameter valuation $\pi$ satisfies a constraint $K$ over the parameters, denoted by $\pi=K$, if the expression obtained by replacing each parameter $\lambda$ in $K$ with $\pi(\lambda)$ evaluates to true. Given two constraints $K_{1}$ and $K_{2}, K_{1}$ is included in $K_{2}$, denoted by $K_{1} \subseteq K_{2}$, if $\forall \pi: \pi=K_{1} \Rightarrow \pi \models K_{2}$. We consider true as a constraint over $\Lambda$, corresponding to the set of all possible values for $\Lambda$.

We denote by $D \downarrow_{\Lambda}$ the constraint over $\Lambda$ obtained by projecting $D$ onto $\Lambda$, i.e. after elimination of the firing times. Formally, $D \downarrow_{\Lambda}=\{\pi \mid \pi \models D\}$.

We finally define intervals as in [TLR09]. An interval $I$ of $\mathbb{R}_{+}$is a $\mathbb{Q}_{+}$-interval if its left endpoint ${ }^{\uparrow} I$ belongs to $\mathbb{Q}_{+}$and its right endpoint $I^{\uparrow}$ belongs to $\mathbb{Q}_{+} \cup$ $\{\infty\}$. We denote by $\mathcal{I}\left(\mathbb{Q}_{+}\right)$the set of $\mathbb{Q}_{+}$-intervals of $\mathbb{R}_{+}$. A parametric time interval is a function $J: \mathbb{Q}_{+}{ }^{\Lambda} \rightarrow \mathcal{I}\left(\mathbb{Q}_{+}\right)$that associates with each parameter valuation a $\mathbb{Q}_{+}$-interval. The set of parametric time intervals over $\Lambda$ is denoted by $\mathcal{J}(\Lambda)$. As for $I$, we define ${ }^{\uparrow} J$ and $J^{\uparrow}$ as the minimum and maximum bounds of $J$, respectively. They can both be represented using a constraint over $\Lambda$.

\subsection{Parametric Time Petri Nets with Inhibitor Arcs}

Parametric time Petri nets with inhibitor arcs (PITPNs) are a parametric extension of ITPNs, where the temporal bounds of the transitions can be parameters. We slightly adapt the notations defined in [TLR09] to fit our setting.

Definition 1. A parametric time Petri nets with inhibitor arcs (PITPN) is a tuple $\mathcal{N}=\left\langle P, T, \Lambda, \bullet(),.(.)^{\bullet},(.)^{\circ}, M_{0}, J_{s}, K_{0}\right\rangle$ where

- $P=\left\{p_{1}, \ldots, p_{m}\right\}$ is a non-empty finite set of places,

- $T=\left\{t_{1}, \ldots, t_{n}\right\}$ is a non-empty finite set of transitions,

$-\Lambda=\left\{\lambda_{1}, \ldots, \lambda_{l}\right\}$ is a finite set of parameters,

- $\bullet$ (.) (resp. (.) $)^{\bullet} \in\left(\mathbb{N}^{P}\right)^{T}$ is the backward (resp. forward) incidence function,

$-(.)^{\circ} \in\left(\mathbb{N}^{P}\right)^{T}$ is the inhibition function,

$-M_{0} \in \mathbb{N}^{P}$ is the initial marking,

- $\left.J_{s} \in \mathcal{J}(\Lambda)\right)^{T}$ is the function that associates a parametric firing interval with each transition, and

- $K_{0} \in \mathcal{L}(\Lambda)$ is the initial constraint over $\Lambda$. 
$K_{0}$ is a constraint over $\Lambda$ giving the initial domain of the parameters, and must at least specify that the minimum bounds of the firing intervals are lower than or equal to the maximum bounds. Additional linear constraints may of course be given. Sometimes, given a constraint $K_{0}$, we will denote a PITPN by $\mathcal{N}\left(K_{0}\right)$ when clear from the context, and to emphasise the value of $K_{0}$ in $\mathcal{N}$.

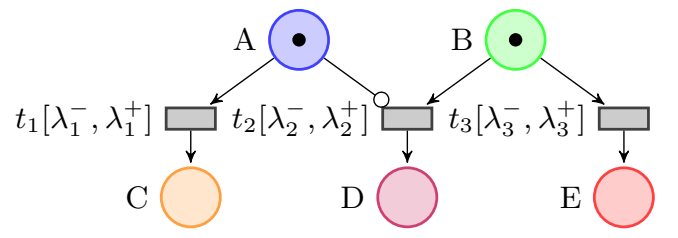

(a) A PITPN $\mathcal{N}$

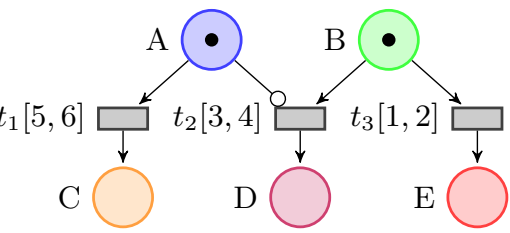

(b) A valuated (P)ITPN $\llbracket \mathcal{N} \rrbracket_{\pi}$

Fig. 2: A PITPN and its valuation

Given a PITPN $\mathcal{N}$ and a valuation $\pi$, we denote by $\llbracket \mathcal{N} \rrbracket_{\pi}$ the (non-parametric) ITPN where each occurrence of a parameter has been replaced by its constant value as in $\pi$. Formally, given $\mathcal{N}=\left\langle P, T, \Lambda,{ }^{\bullet}(),.(.)^{\bullet},(.)^{\circ}, M_{0}, J_{s}, K_{0}\right\rangle$, then $\llbracket \mathcal{N} \rrbracket_{\pi}=\left\langle P, T, \Lambda,{ }^{\bullet}(),.(.)^{\bullet},(.)^{\circ}, M_{0}, J_{s}, K_{0} \wedge K_{\pi}\right\rangle$, where $K_{\pi}=\bigwedge_{\lambda \in \Lambda}(\lambda=\pi(\lambda))$. For example, the ITPN in Fig. 2b corresponds to the PITPN in Fig. 2a valuated with $\pi=\left\{\lambda_{1}^{-} \rightarrow 5, \lambda_{1}^{+} \rightarrow 6, \lambda_{2}^{-} \rightarrow 3, \lambda_{2}^{+} \rightarrow 4, \lambda_{3}^{-} \rightarrow 1, \lambda_{3}^{+} \rightarrow 2\right\}$.

Semantics. We mostly reuse here the definitions and semantics from [TLR09]. The reachable states of a PITPN are parametric state-classes (or simply classes), i.e. pairs $c=(M, D)$ where $M$ is a marking of the net and $D$ is a parametric firing domain, that is, a constraint over $\Theta$ and $\Lambda$. Given a class $c=(M, D)$, a transition $t$ is enabled in $c$ if $M \geq \bullet t$ (i.e. if the number of tokens in $M$ in each input place of $t$ is greater than or equal to the value on the arc between this place and the transition). Transition $t$ is inhibited if the place connected to one of its inhibitor arc is marked with at least as many tokens than the weight of the considered inhibitor arc between this place and $t$. Transition $t$ is active if it is enabled and not inhibited. Transition $t$ is firable if it has been active for at least ${ }^{\uparrow} J_{s}(t)$ time units.

For a given class, the firing times in $\Theta$ correspond to variables encoding the time remaining before an active transition can fire. Hence, these variables decrease with time. The initial class of $\mathcal{N}(K)$ is $c_{0}=\left(M_{0}, D_{0}\right)$, with $D_{0}=$ $K \wedge\left\{\theta_{k} \in J_{s}\left(t_{k}\right) \mid\left(t_{k} \in \operatorname{enabled}\left(M_{0}\right)\right\}\right.$, where $\operatorname{enabled}\left(M_{0}\right)$ denotes the enabled transitions in $M_{0}$. For example, suppose that $K=\lambda_{1}^{-} \leq \lambda_{1}^{+} \wedge \lambda_{2}^{-} \leq \lambda_{2}^{+} \wedge \lambda_{3}^{-} \leq \lambda_{3}^{+}$; then the initial class of $\mathcal{N}$ in Fig. 2a is:

$$
c_{0}=\left(A B, \lambda_{1}^{-} \leq \theta_{1} \leq \lambda_{1}^{+} \wedge \lambda_{2}^{-} \leq \theta_{2} \leq \lambda_{2}^{+} \wedge \lambda_{3}^{-} \leq \theta_{3} \leq \lambda_{3}^{+}\right) .
$$

We consider a (classical) semantics where a transition must fire before its upper interval bound, unless another transition fires first and disables it; for 
example, in Fig. 2a, $t_{1}$ must fire before $t_{3}$ if $\lambda_{1}^{+}<\lambda_{3}^{-}, t_{3}$ must fire before $t_{1}$ if $\lambda_{3}^{+}<\lambda_{1}^{-}$, and both orders are possible otherwise. Given a class $c=(M, D)$ and a firable transition $t_{f}, c^{\prime}=\left(M^{\prime}, D^{\prime}\right)$ can be reached from $c$ in one step via transition $t_{f}$ (denoted by $c \stackrel{t_{f}}{\Rightarrow} c^{\prime}$ ) if the following holds:

$-M^{\prime}=M-\bullet_{f}+t_{f}^{\bullet}$

$-D^{\prime}$ is computed along the following steps:

1. intersection with the firability constraints: $\forall j$ s.t. $t_{j}$ is active, $\theta_{f} \leq \theta_{j}$,

2. variable substitutions for all enabled transitions $t_{j}$ that are active, i.e. $\theta_{j}=\theta_{f}+\theta_{j}^{\prime}$,

3. elimination (using for instance the Fourier-Motzkin method) of all variables relative to transitions disabled by the firing of $t_{f}$,

4. addition of inequalities relative to newly enabled transitions ${ }^{1}: \forall t_{k} \in$ $\operatorname{NewlyEnabled}\left(M, t_{f}\right),{ }^{\uparrow} J_{s}\left(t_{k}\right) \leq \theta_{k}^{\prime} \leq J_{s}\left(t_{k}\right)^{\uparrow}$, with $\operatorname{NewlyEnabled}\left(M, t_{f}\right)$ denoting the set of transitions newly enabled by firing the transition $t_{f}$ from marking $M$.

The full semantics can be found in [TLR09].

A run of $\mathcal{N}$ is a sequence $c_{0} \stackrel{t_{0}}{\Rightarrow} \cdots \stackrel{t_{n}-1}{\Rightarrow} c_{n}$. Given a run $r$ of $\mathcal{N}$ of the form $\left(M_{0}, D_{0}\right) \stackrel{t_{0}}{\Rightarrow} \cdots \stackrel{t_{n}-1}{\Rightarrow}\left(M_{n}, D_{n}\right)$, the trace associated with $r$ is the alternating sequence of markings and actions $M_{0} \stackrel{t_{0}}{\Rightarrow} \ldots \stackrel{t_{n}-1}{\Rightarrow} M_{n}$. The trace set of $\mathcal{N}$ is the set of all traces associated with the runs of $\mathcal{N}$. This corresponds to the discrete (or time-abstract) behaviour of $\mathcal{N}$. Post $_{\mathcal{N}(K)}(C)$ (resp. Post $t_{\mathcal{N}(K)}(C)$ ) is the set of classes reachable from a set $C$ of classes in exactly one step (resp. $i$ steps) in $\mathcal{N}(K)$. Furthermore, we define $\operatorname{Post}_{\mathcal{N}(K)}^{*}(C)$ as $\bigcup_{i>0} \operatorname{Post}_{\mathcal{N}(K)}^{i}(C)$. We define $\operatorname{Reach}(\mathcal{N}(K))$ as the set of reachable classes of $\mathcal{N}(K)$, that is $\operatorname{Post}_{\mathcal{N}(K)}^{*}\left(\left\{c_{0}\right\}\right)$. Finally, we define $\mathcal{G}(\mathcal{N}(K))$ as the parametric reachability graph of $\mathcal{N}(K)$, that is the set of reachable parametric state-classes with the transition relation $\Rightarrow$.

Results. The following lemma, recalled from [TLR09], states that the projection onto the parameters of the constraint associated with a class always gets stronger (i.e. more restricted) along a run of the system.

Lemma 1 (Lemma 14 in [TLR09]). Given a PITPN $\mathcal{N}$, let $c=(M, D)$ and $c^{\prime}=\left(M^{\prime}, D^{\prime}\right)$ be two classes in $\mathcal{G}(\mathcal{N})$. If $c \stackrel{t}{\Rightarrow} c^{\prime}$, then $D^{\prime} \downarrow_{\Lambda} \subseteq D \downarrow_{\downarrow}$.

The following result states that the valuation with $\pi$ of a class $c$ of $\mathcal{N}$ belongs to the graph of $\mathcal{N}$ valuated with $\pi$ if and only if $\pi$ belongs to the constraint associated with $c$.

Theorem 1 (Theorems 12 and 13 in [TLR09]). Given a PITPN $\mathcal{N}(K)$ and a valuation $\pi \models K$, let $c=(M, D)$ be a class in $\mathcal{G}(\mathcal{N}(K))$. Then: $\llbracket c \rrbracket_{\pi} \in$ $\mathcal{G}\left(\llbracket \mathcal{N} \rrbracket_{\pi}\right)$ iff $\pi \models D \downarrow_{\Lambda}$.

\footnotetext{
${ }^{1}$ For sake of simplicity, we only consider here closed intervals of the form $[a, b]$. For open intervals (e.g. $(2,3]$ in Fig. 1a), one should use strict instead of large inequalities.
} 


\section{Covering Constraint}

We introduce the notion of covering constraint as the constraint resulting from the intersection of the projection onto the parameters of the constraints associated with all the reachable classes of a PITPN.

Definition 2. Let $\mathcal{N}$ be a PITPN. The covering constraint of $\mathcal{N}$ is:

$\bigcap_{(M, D) \in \operatorname{Reach}(\mathcal{N})} D \downarrow_{\Lambda}$.

In the general case, it is possible that the covering constraint of a PITPN will be empty, due to the intersection of disjoint constraints over the parameters. But in the setting of the inverse method (see Section 4), it will not be.

The following lemma relates parametric and non-parametric runs, and derives from Theorem 1.

Lemma 2. Let $\mathcal{N}$ be a PITPN, let $\pi$ be a parameter valuation. Let $r$ be a run of $\mathcal{N}$ reaching a class $(M, D)$ in $\mathcal{G}(\mathcal{N})$. Then there exists an equivalent run in $\llbracket \mathcal{N} \rrbracket_{\pi}$ reaching class $\left(M, \llbracket D \rrbracket_{\pi}\right)$ in $\mathcal{G}\left(\llbracket \mathcal{N} \rrbracket_{\pi}\right)$ iff $\pi \models D \downarrow_{\Lambda}$.

Proof. Let $\left(M_{0}, D_{0}\right) \stackrel{t_{0}}{\Rightarrow} \ldots \stackrel{t_{k-1}}{\Rightarrow}\left(M_{k}, D_{k}\right)$ be a run of $\mathcal{N}$. From Theorem 1, we have that $\llbracket\left(M_{k}, D_{k}\right) \rrbracket_{\pi} \in \llbracket \mathcal{G}(\mathcal{N}(K)) \rrbracket_{\pi}$ iff $\pi \models D_{k} \downarrow_{\Lambda}$. Now consider transition $\left(M_{k-1}, D_{k-1}\right) \stackrel{t_{k-1}}{\Rightarrow}\left(M_{k}, D_{k}\right)$ in $\mathcal{G}(\mathcal{N})$. Then, from the semantics of PITPNs, for all $\pi \models \llbracket D_{k} \rrbracket_{\pi}$, then $\left(M_{k-1}, \llbracket D_{k-1} \rrbracket_{\pi}\right) \stackrel{t_{k-1}}{\Rightarrow}\left(M_{k}, \llbracket D_{k} \rrbracket_{\pi}\right) \in \mathcal{G}\left(\llbracket \mathcal{N} \rrbracket_{\pi}\right)$. The result then derives from a reasoning by induction on $k$, with $(M, D)=\left(M_{k}, D_{k}\right)$.

Conversely, the following lemma states that, given a PITPN $\mathcal{N}$, a run in a valuation of $\mathcal{N}$ always has an equivalent run in $\mathcal{N}$.

Lemma 3. Let $\mathcal{N}(K)$ be a PITPN, let $\pi$ be a parameter valuation such that $\pi \models K$. Let $r$ be a run of $\llbracket \mathcal{N} \rrbracket_{\pi}$. Then there exists an equivalent run in $\mathcal{N}(K)$.

Proof. $\llbracket \mathcal{N} \rrbracket_{\pi}$ can be seen as a PITPN (hence parametric) with an initial constraint $K_{\pi}$. Since $K_{\pi} \subseteq K$, from the semantics of PITPNs, the set of behaviours of $\mathcal{N}(K)$ includes the behaviours of $\mathcal{N}\left(K_{\pi}\right)$. Hence any run in $\mathcal{N}\left(K_{\pi}\right)$ has an equivalent in $\mathcal{N}(K)$.

We now state below a general result that will be used to prove Lemma 5 .

Lemma 4. Let $\mathcal{N}(K)$ be a PITPN. Then for all $(M, D) \in \mathcal{G}(\mathcal{N}(K)), D \downarrow_{\Lambda} \subseteq K$.

Proof. By induction on Lemma 1, with $K_{0} \subseteq K$ as the base case.

The following result states that, for a PITPN with its own covering constraint $K_{c o v}$ as initial constraint, the projection onto the parameters of the constraint associated with a reachable class is always the same, and equal to $K_{\operatorname{cov}}$.

Lemma 5. Let $\mathcal{N}(K)$ be a PITPN, let $K_{\text {cov }}$ be the covering constraint of $\mathcal{N}(K)$. Then for all $(M, D) \in \mathcal{G}\left(\mathcal{N}\left(K_{\text {cov }}\right)\right): D \downarrow_{\Lambda}=K_{\text {cov }}$. 
Proof. If $K_{\text {cov }}$ is empty, $\mathcal{G}$ is empty too and the result trivially holds. Suppose $K_{\text {cov }}$ is non-empty. Let $c=(M, D) \in \mathcal{G}\left(\mathcal{N}\left(K_{\text {cov }}\right)\right)$. Let $\pi \models D \downarrow_{\Lambda}$. By Lemma 4, $D \downarrow_{\Lambda} \subseteq K_{\text {cov }}$. By construction of $K_{\text {cov }}$, we have that $K_{\text {cov }} \subseteq K$. Hence $\pi \models$ $D \downarrow_{\Lambda} \Rightarrow \pi \models K$. Since $\pi \models D \downarrow_{\Lambda}$, from Lemma 2, there exists an equivalent run in $\llbracket \mathcal{N} \rrbracket_{\pi}$ reaching class $\left(M, \llbracket D \rrbracket_{\pi}\right)$ in $\mathcal{G}\left(\llbracket \mathcal{N} \rrbracket_{\pi}\right)$. Since $\pi \models K$, from Lemma 3, there exists an equivalent run in $\mathcal{N}(K)$ reaching class $\left(M, D^{\prime}\right)$ for some $D^{\prime}$.

Let $\pi^{\prime} \models K_{\text {cov }}$. By construction, $K_{\text {cov }} \subseteq D^{\prime} \downarrow_{\Lambda}$, hence $\pi^{\prime} \models D^{\prime} \downarrow_{\Lambda}$. By Lemma 4, $D \downarrow_{\Lambda} \subseteq K$, hence $\pi^{\prime}=K$. Since $\pi^{\prime} \models K$ and $\pi^{\prime} \models D^{\prime} \downarrow_{\Lambda}$, applying Theorem 1 to $\mathcal{N}(K)$ gives that $\llbracket c \rrbracket_{\pi^{\prime}} \in \mathcal{G}\left(\llbracket \mathcal{N} \rrbracket_{\pi^{\prime}}\right)$. Since $\pi^{\prime}=K_{\text {cov }}$ by hypothesis, and $\llbracket c \rrbracket_{\pi^{\prime}} \in \mathcal{G}\left(\llbracket \mathcal{N} \rrbracket_{\pi^{\prime}}\right)$, then applying Theorem 1 to $\mathcal{N}\left(K_{\operatorname{cov}}\right)$ gives that $\pi^{\prime} \models D \downarrow_{\Lambda}$. Hence $K_{\text {cov }} \subseteq D \downarrow_{\Lambda}$. (Lemma 4 gives the other direction.)

Finally, Theorem 2 states that the trace set of a PIPTN valuated with any parameter valuation satisfying its covering constraint $K_{\text {cov }}$ is the same as the trace set of this PITPN with $K_{\text {cov }}$ as initial constraint.

Theorem 2. Let $\mathcal{N}$ be a PITPN, let $K_{\text {cov }}$ be the covering constraint of $\mathcal{N}$. Let $\pi \models K_{\text {cov }}$. Then the trace sets of $\mathcal{N}\left(K_{\text {cov }}\right)$ and $\llbracket \mathcal{N} \rrbracket_{\pi}$ are equal.

Proof. Let $\pi \mid=K_{\text {cov }}$. Consider a run of $\mathcal{N}\left(K_{\text {cov }}\right)$ reaching a class $(M, D)$ in $\mathcal{G}\left(\mathcal{N}\left(K_{\text {cov }}\right)\right)$. By Lemma 5 , it holds that $D \downarrow_{\Lambda}=K_{\text {cov }}$. Since $\pi \models K_{\text {cov }}$, then $\pi \models D \downarrow_{\Lambda}$. Hence, by Lemma 2 , there exists an equivalent run in $\mathcal{G}\left(\llbracket \mathcal{N} \rrbracket_{\pi}\right)$. Conversely, since $\pi=K_{\text {cov }}$, by lemma 3 , any run in $\llbracket \mathcal{N} \rrbracket_{\pi}$ has an equivalent run in $\mathcal{N}\left(K_{\text {cov }}\right)$.

We can derive from Theorem 2 that the trace set of a PIPTN with any parameter valuation satisfying its covering constraint is always the same. This result will be used to prove the correctness of the inverse method (see Section 4).

Corollary 1. Let $\mathcal{N}$ be a PITPN, let $K_{\text {cov }}$ be the covering constraint of $\mathcal{N}$. Then for all $\pi, \pi^{\prime} \models K_{\text {cov }}$, the trace sets of $\llbracket \mathcal{N} \rrbracket_{\pi}$ and $\llbracket \mathcal{N} \rrbracket_{\pi^{\prime}}$ are equal.

\section{The Inverse Method for Time Petri Nets}

We extend to PITPNs the inverse method initially proposed for timed automata [AS13]. The algorithm relies on the following definition of $\pi$-compatibility.

Definition 3. Given a parameter valuation $\pi$, a class $(M, D)$ is said to be $\pi$ compatible if $\pi \models D \downarrow_{\Lambda}$, and $\pi$-incompatible otherwise.

\subsection{Principle}

We introduce in Algorithm 1 IMPN (i.e. the Inverse Method for time Petri Nets with inhibitor arcs). It uses 3 variables: an integer $i$ measuring the depth of the state space exploration, the current constraint $K_{c}$, and the set $C$ of explored classes. Starting from the initial class $c_{0}, I M P N$ iteratively computes 


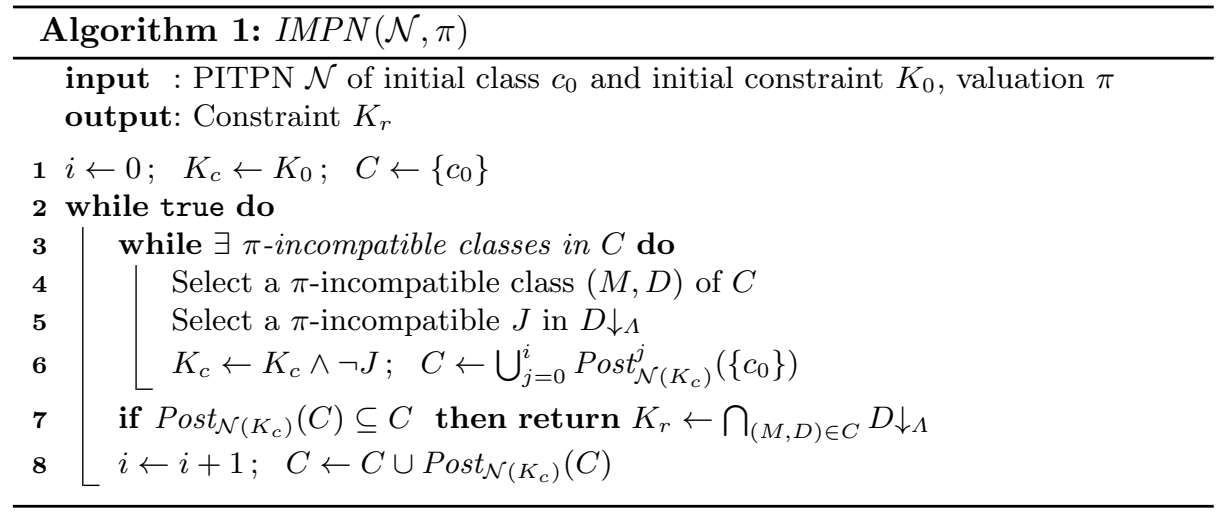

classes. When a $\pi$-incompatible class is found, an incompatible inequality is nondeterministically selected within the projection of the constraint onto $\Lambda$ (line 5); its negation is then added to $K_{c}$ (line 6 ). The set of reachable classes is then updated. When all successor classes have already been reached (line 7), IMPN returns the intersection $K_{r}$ of the projection onto $\Lambda$ of the constraints associated with all the reachable classes.

\section{$4.2 \quad$ Results}

Lemma 6. Let $\mathcal{N}$ be a PITPN, and $\pi$ be a parameter valuation. Suppose that algorithm $\operatorname{IMPN}(\mathcal{N}, \pi)$ terminates with output $K_{r}$. It holds that $\pi \models K_{r}$.

Proof. By construction, at the end of the inner while loop, all classes of $C$ are $\pi$-compatible, that is for all $(M, D) \in C, \pi \models D$. As a consequence, $\pi \models D \downarrow_{\Lambda}$. Recall that $K_{r}=\bigcap_{(M, D) \in C} D \downarrow_{\Lambda}$. Hence $\pi \models K_{r}$.

The correctness of IMPN mainly relies on the fact that $K_{r}$ is the covering constraint of $\mathcal{N}$. Hence, the results of Section 3 can be applied.

Theorem 3 (Correctness). Let $\mathcal{N}$ be a PITPN, and $\pi$ be a parameter valuation. Suppose $\operatorname{IMPN}(\mathcal{N}, \pi)$ terminates with output $K_{r}$. Then:

1. $\pi \models K_{r}$, and

2. $\forall \pi^{\prime} \models K_{r}, \llbracket \mathcal{N} \rrbracket_{\pi^{\prime}}$ and $\llbracket \mathcal{N} \rrbracket_{\pi}$ have the same trace set.

Proof. Item 1 comes from Lemma 6 . For item 2 , since $K_{r}$ is the covering constraint of $\mathcal{N}$, then we can apply Corollary 1 , which gives the result. Also note that the covering constraint cannot be empty since $\pi \models K_{r}$.

Non-termination. Parameter synthesis is undecidable for PITPNs [TLR09] and $I M P N$ may not always terminate. Consider the PITPN $\mathcal{N}$ in Fig. 3a; then, $I M P N$ applied to $\mathcal{N}$ and a reference valuation with all parameters equal to 0 will generate an infinite set of classes with constraints of the form $i * \lambda_{1}^{-} \leq \lambda_{2}^{+}$, 


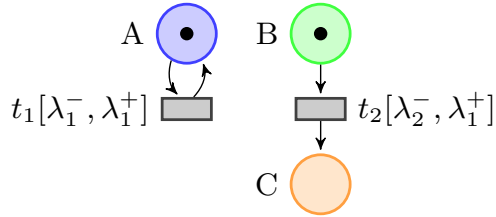

(a) Non-termination

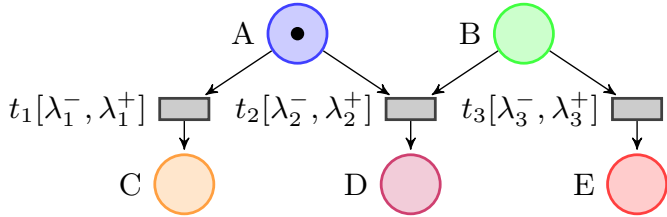

(b) Non-completeness

Fig. 3: Counter-examples PITPNs

with $i$ infinitely growing. Intuitively, $t_{1}$ can fire an arbitrary number of times before $t_{2}$ fires. Of course, this is a typical Zeno-behaviour (an infinite number of transitions within a null duration) and, in the case of non-null reference parameter valuations, an inequality $i * \lambda_{1}^{-} \leq \lambda_{2}^{+}$will eventually be $\pi$-incompatible, thus ensuring termination. Also note $\mathcal{N}$ is a bounded $\mathrm{L} / \mathrm{U}$ (lower/upper bounds) PTPN [TLR09], showing that termination of IMPN is not guaranteed for general bounded L/U PTPNs (although emptiness and reachability problems are decidable in theory). Studying the decidability of this problem, and adapting IMPN to ensure termination in this case is the subject of ongoing work.

We can exhibit subclasses for which IMPN terminates. This is obviously the case of loopless PITPNs (in which no syntactical loop exists in the model). This is also the case of parametric sequential TPNs [AHJR12]; this subclass of TPNs is such that each time a discrete transition is fired, each transition that is enabled in the new/resulting marking is newly enabled. Hence, the problem of infinitely concurrent loops such as in Fig. 3a cannot happen.

Non-confluence and Non-completeness. Due to the non-deterministic selection of an inequality, IMPN is non-confluent (i.e. different applications of the algorithm can yield different outputs). As a consequence, it is also non-complete (i.e. the resulting constraint may not be the maximal one). Formally:

Proposition 1 (Non-completeness). There may exist $\pi^{\prime} \not \models K_{r}$ such that $\llbracket \mathcal{N} \rrbracket_{\pi^{\prime}}$ and $\llbracket \mathcal{N} \rrbracket_{\pi}$ have the same trace set.

An example for non-completeness is the PITPN $\mathcal{N}$ in Fig. 3b, with the reference parameter valuation $\pi=\left\{\lambda_{1}^{-} \rightarrow 5, \lambda_{1}^{+} \rightarrow 6, \lambda_{2}^{-} \rightarrow 1, \lambda_{2}^{+} \rightarrow 3, \lambda_{3}^{-} \rightarrow 2, \lambda_{3}^{+} \rightarrow 4\right\}$. In $\llbracket \mathcal{N} \rrbracket_{\pi}$, either $t_{2}$ or $t_{3}$ can fire first, but not $t_{1}$, due to the fact that we have both ${ }^{\uparrow} I\left(t_{1}\right)>I^{\uparrow}\left(t_{2}\right)$ and ${ }^{\uparrow} I\left(t_{1}\right)>I^{\uparrow}\left(t_{3}\right)$.

When applying the inverse method to $\mathcal{N}$ and $\pi$, a class will be generated with $\mathrm{BC}$ as a marking, and an associated constraint containing in particular inequalities $\lambda_{1}^{-} \leq \lambda_{2}^{+} \wedge \lambda_{1}^{-} \leq \lambda_{3}^{+}$. Since both are $\pi$-incompatible, the algorithm can add to the current constraint either $\lambda_{1}^{-}>\lambda_{2}^{+}$or $\lambda_{1}^{-}>\lambda_{3}^{+}$. Either of them is sufficient to prevent $\mathrm{BC}$ to be reachable. Then the result of the application of IMPN to $\mathcal{N}$ and $\pi$ is both non-confluent and non-complete. Also note that, due to the absence of inhibitor arc in $\mathcal{N}$, the non-completeness of IMPN also holds for PTPNs. 
Nevertheless, it can be shown (as it was the case for timed automata [AS13]) that a sufficient (but non-necessary) condition for completeness is that IMPN does not perform non-deterministic selections of inequalities, i.e. at most one $\pi$-incompatible class is met at each iteration.

\section{Precise Robustness Analysis}

\subsection{Local Robustness}

Throughout this section, we assume an ITPN $N$, as well as a parameterised version $\mathcal{N}$ of $N$ where each lower (resp. upper) bound of a transition $t_{i}$ is replaced with a fresh parameter $\lambda_{i}^{-}\left(\operatorname{resp} . \lambda_{i}^{+}\right)$. Let $\pi$ be the reference valuation such that $\llbracket \mathcal{N} \rrbracket_{\pi}=N$. We assume that $\operatorname{IMPN}(\mathcal{N}, \pi)$ terminates with output $K_{r}$.

We will exploit $K_{r}$ to characterise the precise robustness of the system, i.e. the admissible variability of each timing bound. The original trace set is preserved by any valuation satisfying $K_{r}$. Hence, any linear-time (LTL) property that is true in $\llbracket \mathcal{N} \rrbracket_{\pi}$ is also true in $\llbracket \mathcal{N} \rrbracket_{\pi^{\prime}}$, for $\pi^{\prime} \models K_{r}$. Thus, if the correctness is given in the form of an LTL property, the timing delays can safely vary as long as they satisfy $K_{r}$.

We use here several examples in order to better illustrate the notions. For the PITPN in Fig. 2a, with $\pi=\left\{\lambda_{1}^{-} \rightarrow 5, \lambda_{1}^{+} \rightarrow 6, \lambda_{2}^{-} \rightarrow 3, \lambda_{2}^{+} \rightarrow 4, \lambda_{3}^{-} \rightarrow\right.$ $\left.1, \lambda_{3}^{+} \rightarrow 2\right\}$ as a reference valuation, IMPN outputs the constraint $K_{r}=\lambda_{1}^{-} \leq$ $\lambda_{1}^{+} \wedge \lambda_{2}^{-} \leq \lambda_{2}^{+} \wedge \lambda_{3}^{-} \leq \lambda_{3}^{+} \wedge \lambda_{3}^{+}<\lambda_{1}^{-}$. For a parameterised version of the ITPN in Fig. 1a, IMPN outputs the constraint $K_{r}=\lambda_{1}^{-} \leq \lambda_{1}^{+} \wedge \lambda_{2}^{-} \leq \lambda_{2}^{+} \wedge \lambda_{2}^{-} \geq \lambda_{1}^{+}$. For a parameterised version of the ITPN in Fig. 1b, IMPN outputs the constraint $K_{r}=\lambda_{1}^{-} \leq \lambda_{1}^{+} \wedge \lambda_{2}^{-} \leq \lambda_{2}^{+} \wedge \lambda_{3}^{-}=0 \wedge 0 \leq \lambda_{3}^{+} \wedge \lambda_{1}^{+}=\lambda_{2}^{-}$.

Definition 4. An ITPN $N$ is robust with respect to linear-time properties (or LT-robust) if there exists $\gamma>0$ such that for any linear time property $\varphi, N^{\prime} \models \varphi$ if and only if $N \models \varphi$, where $N^{\prime}$ is an ITPN similar to $N$ where each timing bound $c$ can be replaced with any value within $[c-\gamma, c+\gamma]$.

For example, the ITPN in Fig. 2a is LT-robust (with e.g. $\gamma=1$ ), whereas the ITPNs in Fig. 1 are not.

Local Robustness. The resulting constraint $K_{r}$ is given in the form of a convex (possibly unbounded) polyhedron. For each interval bound $\lambda_{i}$ in $\mathcal{N}$, its local robustness $\operatorname{LR}\left(\lambda_{i}\right)$ is defined as the distance between $\pi\left(\lambda_{i}\right)$ and the closest border of the polyhedral representation of $K_{r}$. For example, in Fig. 2a, $L R\left(\lambda_{1}^{-}\right)=1$. In Fig. 1a, $L R\left(\lambda_{1}^{-}\right)=1$ whereas $L R\left(\lambda_{1}^{+}\right)=0$, showing that this latter bound renders the system non-robust. The following lemma follows from Definition 4, from the definition of $L R$ and the correctness of $I M P N$.

Lemma 7. If for each parameter $\lambda$ in $\mathcal{N}, L R(\lambda)>0$, then $N$ is LT-robust.

Ranging Interval. For each interval bound $\lambda_{i}$ in $\mathcal{N}$, its ranging interval $R I\left(\lambda_{i}\right)$ is defined as its minimum and maximum admissible values within $K_{r}$. It is 
computed by valuating all parameters but $\lambda_{i}$ in $K_{r}$, and converting the resulting inequality in the form of an interval. For example, in Fig. $2 \mathrm{a}, R I\left(\lambda_{1}^{-}\right)=(2,6]$. In Fig. 1a, $R I\left(\lambda_{1}^{+}\right)=[1,2]$.

The local lower (resp. upper) variability is defined as the distance between the parameter valuation and the lower (resp. upper) bound of $R I$; formally, given $R I\left(\lambda_{i}\right)=(a, b), L L V\left(\lambda_{i}\right)=\pi\left(\lambda_{i}\right)-a$ and $L U V\left(\lambda_{i}\right)=b-\pi\left(\lambda_{i}\right)$. Note that the local robustness can be obtained from the local variability: $L R\left(\lambda_{i}\right)=$ $\min \left(L L V\left(\lambda_{i}\right), L U V\left(\lambda_{i}\right)\right)$.

Computation of $\Delta$. Our approach also allows to retrieve the value of the " $\Delta$ " of $\Delta$-based approaches. It is defined as the minimum over the set of parameters of the distance between a parameter and the closest border of the polyhedron. Formally, $\Delta=\min \left(\min _{i \in \Delta^{-}} L L V\left(\lambda_{i}\right), \min _{i \in \Delta^{+}} L U V\left(\lambda_{i}\right)\right)$, where $\Delta^{-}\left(\right.$resp. $\left.\Delta^{+}\right)$ denotes the set of parameters appearing in an interval lower (resp. upper) bound. This distinction is necessary, since $\Delta$-based approaches only consider the positive enlarging of intervals. For the ITPN in Fig. 2a, the maximum possible $\Delta$ is 1.5 (see Section 5.3). And, obviously, $\Delta=0$ for the ITPNs in Fig. 1.

\subsection{Improving the System Robustness}

Identifying Critical Timing Bounds. Our approach allows to exhibit critical timing bounds: critical timing bounds are those rendering the system non-robust, i.e. with a null local robustness. For example, in Fig. $1 \mathrm{a}, \lambda_{1}^{+}$and $\lambda_{2}^{-}$are the critical timing bounds. In Fig. $1 \mathrm{~b}, \lambda_{1}^{+}, \lambda_{2}^{-}$and $\lambda_{3}^{-}$are the critical timing bounds.

Relaxing Bounds. For some systems, it is possible to refine the values of the critical timing bounds so that the system becomes robust, with the same discrete behaviour. In practice, this may in particular be the case of hardware systems, where the timing bounds come from the traversal time of micro components: One can change the timing bounds by replacing a component with another one. In software, one can also refine the values of some timers if needed.

In that case, one can exploit the precise robustness analysis to synthesise values for the timing bounds so that the system is robust. A system is said to be potentially robust if all timing bounds $\lambda_{i}$ have a ranging interval non-reduced to a point (even if their local robustness may possibly be null, i.e. $L R\left(\lambda_{i}\right)=0$ ).

Definition 5. An ITPN $N$ is potentially robust if, for all timing bounds $\lambda_{i}$, $L L V\left(\lambda_{i}\right)>0$ or $L U V\left(\lambda_{i}\right)>0$.

This notion of potential robustness is a sufficient condition so that an ITPN becomes robust with the same discrete behaviour.

Theorem 4. If $N$ is potentially robust, then there exists $\pi_{R}$ such that $\llbracket \mathcal{N} \rrbracket_{\pi_{R}}$ is LT-robust, and has the same trace set as $N$.

Proof. By Lemma 7, only the timing bounds $\lambda_{i}$ such that $L R\left(\lambda_{i}\right)=0$ render $\mathcal{N}$ non-LT-robust. For all $\lambda_{i}$ such that $L R\left(\lambda_{i}\right)>0$, we set $\pi_{R}\left(\lambda_{i}\right)=\pi\left(\lambda_{i}\right)$. Now consider a $\lambda_{i}$ such that $L R\left(\lambda_{i}\right)=0$. By definition of $L R$, either $L L V\left(\lambda_{i}\right)=0$ 
or $\operatorname{LUV}\left(\lambda_{i}\right)=0$. Consider the former case (the latter case is dual). Let $(a, b)=$ $R I\left(\lambda_{i}\right)$. Let $\left.\pi_{R}\left(\lambda_{i}\right)=\pi\left(\lambda_{i}\right)+(a+b)\right) / 2$. Since $\mathcal{N}$ is potentially robust, and since $L L V\left(\lambda_{i}\right)=0$, then $L U V\left(\lambda_{i}\right)>0$. By definition of $L U V, L U V\left(\lambda_{i}\right)=b-\pi\left(\lambda_{i}\right)$, hence $b>\pi\left(\lambda_{i}\right)$. Hence $a<\pi_{R}\left(\lambda_{i}\right)<b$. As a consequence, in $\pi_{R}$, we have $L R\left(\lambda_{i}\right)>0$. By construction, and from the convexity of $K_{r}, \pi_{R}\left(\lambda_{i}\right)$ is in $K_{r}$; hence, from Theorem $3, \llbracket \mathcal{N} \rrbracket_{\pi_{R}}$ and $\llbracket \mathcal{N} \rrbracket_{\pi}$ have the same trace set.

Note that this is a sufficient but non-necessary condition, since the notion of potential robustness is based on $L L V$ and $L U V$, that come from $K_{r}$, which is non-complete. Furthermore, one can find further conditions (and constructions) to render a system robust. For example, the ITPN in Fig. 1b is not potentially robust; but it can be made robust with the same discrete behaviour, e.g. by replacing the intervals associated with both $t_{1}$ and $t_{2}$ with $[0,1]$.

\subsection{Comparison with $\Delta$-based Approaches}

The main drawback of our approach is that it does not terminate in the general case, although we exhibited cases for which termination is guaranteed (see Section 4.2). In contrast, related work show that deciding only whether a system is robust is decidable in most cases. However, beside the fact that we give a quantitative measure of the robustness in the form of a constraint in $2 * n$ dimensions (with $n$ the number of transitions), our approach is particularly interesting in the case of a non-robust system. First, we exhibit which timing bounds are responsible for the non-robustness. Second, we give a condition to render the system robust without changing its discrete behaviour.

Furthermore, our approach may output a significantly larger constraint than the $\Delta$-cube output by $\Delta$-based approaches. Actually, when the result of IMPN is complete, the resulting polyhedron is necessarily at least as large as the $\Delta$-cube. Consider again the example in Fig. 2a. In order to enable a graphical comparison in 2 dimensions, we assign all parameters but $\lambda_{1}^{-}$and $\lambda_{3}^{+}$to their value as in $\pi$. Hence the constraint becomes $\lambda_{1}^{-} \leq 6 \wedge 1 \leq \lambda_{3}^{+} \wedge \lambda_{3}^{+}<\lambda_{1}^{-}$. This constraint is depicted in Fig. 4a. As of $\Delta$-based approaches, they cannot compute a value for $\Delta$ greater than 1.5 in this situation. Indeed, with $\Delta=1.5, \lambda_{3}^{+}$becomes $\lambda_{3}^{+}+\Delta=3.5, \lambda_{1}^{-}$becomes $\lambda_{1}^{-}-\Delta=3.5$, in which case the discrete behaviour becomes different $\left(t_{1}\right.$ can fire before $\left.t_{3}\right)$. This $\Delta$ is given in Fig. $4 \mathrm{~b}$.

The interpretation of the much larger parametric domain covered by $K_{r}$ compared to the $\Delta$-cube can be explained as follows: (1) The parametric domain below $\lambda_{3}^{+}=2$ and above $\lambda_{1}^{-}=5$ is not covered by the $\Delta$-cube, because $\Delta$-based approaches consider a positive parameter $\Delta \geq 0$. Hence, it is not possible to study, e.g. by how much an upper bound can be decreased. (2) The constraint $K_{r}$ allows to relate parameters. Whereas the value of $\Delta$ prevents $\lambda_{1}^{-}$and $\lambda_{3}^{+}$to vary by more than 1.5 , the inequality $\lambda_{3}^{+}<\lambda_{1}^{-}$states that $\lambda_{1}^{-}$may vary by more than 1.5, as long as $\lambda_{3}^{+}$varies less (i.e. $\lambda_{3}^{+}<\lambda_{1}^{-}$). This is of particular interest in systems where some bounds are more likely to vary than others. (3) This small example is a "good" example for $\Delta$-based approaches. In the case where at least one parameter cannot vary, $\Delta$ would be inevitably equal to 0 , whereas $K_{r}$ would still give an output for other dimensions. This is the case of the ITPNs in Fig. 1. 


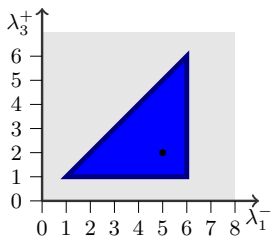

(a) Representation of $K_{r}$

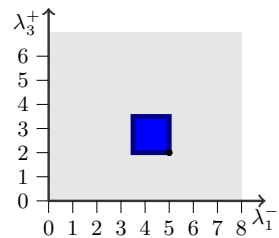

(b) Representation of the $\Delta$-cube

Fig. 4: Graphical comparison for the example in Fig. 2a

\section{Final Remarks}

In this paper, we extended the inverse method to PITPNs and showed how to exploit its output to obtain a precise quantitative measure of the system robustness for linear-time properties. This paper considers the quantification of the system robustness with respect to linear-time (hence time-abstract) properties only. Nevertheless, timed properties can also be considered, by adding an observer net. This observer synchronises with the system ITPN, and can reduce timed properties to time-abstract properties.

Our algorithms should be implemented and compared with similar tools, such as Shrinktech [San13]. Finally, we only addressed here the variability of the timing delays $(\Delta)$, but not the admissible variations of the clock speed (usually called " $\epsilon$ "). Our approach could be extended to this setting using extensions of the inverse method for parameterised hybrid systems [FK13], by adding for each

clock two additional parameters $\epsilon_{i}^{-}$and $\epsilon_{i}^{+}$measuring the admissible decrease and increase speed rate.

Acknowledgment. We are grateful to an anonymous reviewer for his/her very detailed comments.

\section{References}

AD94. Rajeev Alur and David L. Dill. A theory of timed automata. Theoretical Computer Science, 126(2):183-235, 1994.

$\mathrm{AHJ}^{+}$12. S. Akshay, Loïc Hélouët, Claude Jard, Didier Lime, and Olivier H. Roux. Robustness of time Petri nets under architectural constraints. In FORMATS, volume 7595 of Lecture Notes in Computer Science, pages 11-26. Springer, 2012.

AHJR12. S. Akshay, Loïc Hélouët, Claude Jard, and Pierre-Alain Reynier. Robustness of time Petri nets under guard enlargement. In RP, volume 7550 of Lecture Notes in Computer Science, pages 92-106. Springer, 2012.

AS13. Étienne André and Romain Soulat. The Inverse Method. FOCUS Series in Computer Engineering and Information Technology. ISTE Ltd and John Wiley \& Sons Inc., 2013. 
$\mathrm{BLM}^{+}$11. Patricia Bouyer, Kim G. Larsen, Nicolas Markey, Ocan Sankur, and Claus R. Thrane. Timed automata can always be made implementable. In CONCUR, volume 6901 of Lecture Notes in Computer Science, pages 76-91. Springer, 2011.

BMS12. Patricia Bouyer, Nicolas Markey, and Ocan Sankur. Robust reachability in timed automata: A game-based approach. In ICALP, volume 7392 of Lecture Notes in Computer Science, pages 128-140. Springer, 2012.

FK13. Laurent Fribourg and Ulrich Kühne. Parametric verification and test coverage for hybrid automata using the inverse method. IJFCS, 24(2):233-249, 2013.

JR11. Rémi Jaubert and Pierre-Alain Reynier. Quantitative robustness analysis of flat timed automata. In FoSSaCS, volume 6604 of Lecture Notes in Computer Science, pages 229-244. Springer-Verlag, 2011.

Mar11. Nicolas Markey. Robustness in real-time systems. In SIES, pages 28-34. IEEE Computer Society Press, 2011.

Mer74. Philip Meir Merlin. A study of the recoverability of computing systems. PhD thesis, University of California, Irvine, CA, USA, 1974.

San13. Ocan Sankur. Shrinktech: A tool for the robustness analysis of timed automata. In $C A V$, Lecture Notes in Computer Science. Springer, 2013. To appear.

TLR09. Louis-Marie Traonouez, Didier Lime, and Olivier H. Roux. Parametric model-checking of stopwatch Petri nets. Journal of Universal Computer Science, 15(17):3273-3304, 2009.

Tra12. Louis-Marie Traonouez. A parametric counterexample refinement approach for robust timed specifications. In FIT, volume 87 of EPTCS, pages 17-33, 2012. 